\title{
HOMOGENEOUS QUASI-INVARIANT SUBSPACES OF THE FOCK SPACE
}

\author{
KUNYU GUO \\ (Received 12 April 2001; revised 30 October 2002) \\ Communicated by G. Willis
}

\begin{abstract}
In this paper, we prove that two homogeneous quasi-invariant subspaces are similar only if they are equal. Moreover, we exhibit an example to show how to determine the similarity orbits of quasi-invariant subspaces.
\end{abstract}

2000 Mathematics subject classification: primary 46J15, 46H25, 47A15.

Keywords and phrases: the Fock space, quasi-invariant subspace, similarity orbit, quasi-module map.

\section{Introduction}

Recall that the Hardy space $H^{2}(\mathbb{D})$ over the open unit disk $\mathbb{D}$ is the closed subspace of $L^{2}(\mathbb{T})$ spanned by the non-negative powers of the coordinate function $z$. If $M$ is a (closed) subspace of $H^{2}(\mathbb{D})$ that is invariant for the multiplication operator $M_{2}$, then Beurling's theorem says that there exists an inner function $\eta$ such that $M=\eta H^{2}(\mathbb{D})$. Beurling's theorem has played an important role in operator theory, function theory and their intersection, function-theoretic operator theory. Let $\Omega$ be a bounded domain in $\mathbb{C}^{n}$, and let $X$ be a Hilbert space consisting of analytic functions in $\Omega$ such that $1 \in X$, and for each polynomial $p$ and each $h \in X, p h \in X$. If $M$ is a closed subspace of $X$ such that $p M \subseteq M$ for every polynomial $p$, we say that $M$ is an invariant subspace for the function space $X$.

Despite the great development in these fields over the past fifty years, there are still many problems to explore, one of which is to investigate equivalence classes of invariant subspaces of function spaces under similarity or unitary equivalence.

Supported by NNSFC(10171019), Shuguang project in Shanghai and Young teacher Fund of higher school of National Educational Ministry of China.

(C) 2003 Australian Mathematical Society 1446-7887/03 \$A2.00+0.00 
Along this line, Axler, Agrawal, Bourdon, Douglas, Guo, Paulsen, Putinar, Salinas have done a lot of work, see $[1,2,4,7-12,14,15]$ and references therein. The extension of some of the above results to the Fock space, and analytic Hilbert spaces on the complex plane were considered by Guo and Zheng [13], and by Chen, Guo and Hou [6].

The Fock space is the analog of the Bergman space in the context of the complex $n$-space $\mathbb{C}^{n}$. It is a Hilbert space consisting of entire functions in $\mathbb{C}^{n}$. Let

$$
d \mu(z)=e^{-|z|^{2} / 2} d v(z)(2 \pi)^{-n}
$$

be the Gaussian measure on $\mathbb{C}^{n}$ ( $d v$ is the ordinary Lebesgue measure). The Fock space $L_{a}^{2}\left(\mathbb{C}^{n}, d \mu\right)$ (for short, $L_{a}\left(\mathbb{C}^{n}\right)$ ), by definition, is the space of all $\mu$-square-integrable entire functions on $\mathbb{C}^{n}$. It is easy to see that $L_{a}^{2}\left(\mathbb{C}^{n}\right)$ is a closed subspace of $L^{2}\left(\mathbb{C}^{n}\right)$ with the reproducing kernel functions $K_{\lambda}(z)=e^{\bar{\lambda} z / 2}$, and the normalized reproducing kernel functions $k_{\lambda}(z)=e^{\bar{\lambda} z / 2-|\lambda|^{2} / 4}$, (here $\bar{\lambda} z=\sum_{i=1}^{n} \bar{\lambda}_{i} z_{i}$ ).

The next proposition states that there exists no nontrivial invariant subspace for the Fock space. This proposition first appeared in [13].

PROPOSITION 1.1. Let $M$ be a (closed) subspace of $L_{a}^{2}\left(\mathbb{C}^{n}\right)$, and $M \neq\{0\}$. If $f$ is an entire function on $C^{n}$ such that $f M \subset M$, then $f$ is a constant.

Thus, an appropriate substitute for invariant subspace, the so-called quasi-invariant subspace is needed. Namely, a (closed) subspace $M$ of the Fock space is called quasiinvariant if the relation $p f \in L_{a}^{2}\left(\mathbb{C}^{n}\right)$ implies $p f \in M$ for any $f \in M$ and any polynomial $p$. Equivalently, $M$ is quasi-invariant if $p M \cap L_{a}^{2}\left(\mathbb{C}^{n}\right) \subset M$ for each polynomial $p$.

Let $M_{1}$ and $M_{2}$ be two quasi-invariant subspaces of $L_{a}^{2}\left(\mathbb{C}^{n}\right)$. For a bounded linear operator $A: M_{1} \rightarrow M_{2}$, we call $A$ a quasi-module map if $A(p f)=p A(f)$ whenever $p f \in M_{1}$ (here $p$ is any polynomial, and $f \in M_{1}$ ). Thus by the definition if $A$ is a quasi-module map, then the relation $p f \in M_{1}$ forces $p A(f) \in M_{2}$. Letting $M_{1}$ and $M_{2}$ be quasi-invariant subspaces of $L_{a}^{2}\left(\mathbb{C}^{n}\right)$, we say that

(1) they are unitarily equivalent if there exists a unitary quasi-module map $A$ : $M_{1} \rightarrow M_{2}$ such that $A^{-1}: M_{2} \rightarrow M_{1}$ is also quasi-module map;

(2) they are similar if there exists an invertible quasi-module map $A: M_{1} \rightarrow M_{2}$ such that $A^{-1}: M_{2} \rightarrow M_{1}$ is also a quasi-module map.

It is easy to check that unitary equivalence and similarity are equivalence relations in the category of all quasi-invariant subspaces.

It is well known that for each analytic function space $X$ on a bounded domain $\Omega$, the closure $\bar{I}$ of an ideal $I$ of polynomial ring $\mathscr{C}$ is an invariant subspace of $X$. However it is never obvious if the closure $\bar{I}$ of $l$ in the Fock space $L_{a}^{2}\left(\mathbb{C}^{n}\right)$ is quasi-invariant. In Section 2 , we first prove that the closure of a homogeneous ideal is quasi-invariant. 
Furthermore, it is shown that two homogeneous quasi-invariant subspaces are similar only if they are equal. In Section 3, we determine the similarity orbit of the quasiinvariant subspace $\left[z^{m}\right]$ generated by $z^{m}$. Namely, the similarity orbit of $\left[z^{m}\right]$ consists of $[p(z)]$, where $p(z)$ range over all polynomials in the variable $z$ with $\operatorname{deg} p=m$.

\section{Homogeneous quasi-invariant subspaces of the Fock space}

It is well known that for each analytic Hilbert space $X$ on a bounded domain $\Omega$, the invariant subspace generated by an ideal $I$ of polynomial ring $\mathscr{C}$ is the closure $\bar{I}$ of $I$. However, in the case of the Fock space it is never obvious if the quasi-invariant subspace generated by $I$ is the closure $\bar{I}$ of $I$. Here we give a proposition which shows that the quasi-invariant subspace generated by a homogeneous ideal is the closure of this ideal. Recall that an ideal $I$ is homogeneous if the relation $p \in I$ implies that all homogeneous components of $p$ are in $I$. Equivalently, an ideal $I$ is homogeneous if and only if $I$ is generated by homogeneous polynomials.

Proposition 2.1. Let I be a homogeneous ideal. Then on the Fock space $L_{a}^{2}\left(\mathbb{C}^{n}\right)$, the quasi-invariant subspace generated by $I$ is the closure $\bar{I}$ of $I$.

PROOF. Let $f \in \bar{I}$, and $f=\sum_{k=0}^{\infty} f_{k}$ be $f^{\prime}$ s homogeneous expression. We claim that every $f_{k}$ is in $I$. To prove the claim, we let $\mathscr{I}_{k}$ consist of all those $p \in I$ with homogeneous degree of $p$ being at most $k$. Then $\mathscr{I}_{k}$ is of finite dimension. From the relation $f \in \bar{l}$, there is a sequence $\left\{p_{m}\right\}$ in $I$ such that $p_{m} \rightarrow f$ as $m \rightarrow \infty$. This implies that $p_{m}^{(k)} \rightarrow f_{k}$, where $p_{m}^{(k)}$ denote $k$-homogeneous component of $p_{m}$. Since $I$ is homogeneous, $p_{m}^{(k)}$ belong to $I$, and hence they are in $\mathscr{I}_{k}$. Because $\mathscr{I}_{k}$ is finite dimensional, and hence closed, this forces $f_{k} \in I$.

Assume that $q f \in L_{a}^{2}\left(\mathbb{C}^{n}\right)$ for some polynomial $q$. Let $q=\sum_{i=0}^{l} q_{i}$ be the homogeneous expression of $q$. Then the homogeneous expression of $q f$ is given by

$$
q f=\sum_{m=0}^{\infty}\left(\sum_{i+j=m} q_{i} f_{j}\right) .
$$

Now it is easy to derive that $q f \in \bar{I}$ by the above homogeneous expression of $q f$. It follows that $\bar{I}$ is quasi-invariant, and hence it equals the quasi-invariant subspace generated by $I$.

THEOREM 2.2. Let $I_{1}$ and $I_{2}$ be homogeneous ideals. Then $\overline{I_{1}}$ and $\overline{I_{2}}$ are similar if and only if $I_{1}=I_{2}$.

To prove theorem we need some preliminaries. For a polynomial $p$, we use $\operatorname{deg} p$ to denote the homogeneous degree of $p$. First we give the following proposition. 
PROPOSITION 2.3. Let $A: M_{1} \rightarrow M_{2}$ be a quasi-module map. Then $A$ maps $M_{1} \cap \mathscr{C}$ to $M_{2} \cap \mathscr{C}$. Furthermore, if $p \in M_{1} \cap \mathscr{C}$, then $\operatorname{deg} p \geq \operatorname{deg} A(p)$.

PROOF. We may assume that $M_{1}$ contains a nonzero polynomial $p$. Set $q=A(p)$. We claim that $\operatorname{deg} q \leq \operatorname{deg} p$. Let $p=\sum_{k=0}^{\operatorname{deg} p} p_{k}, q=\sum_{k=0}^{\infty} q_{k}$ be the homogeneous expansions of $p$ and $q$, respectively. Then for each positive integer $N$, one has

$$
\left\|\left(z_{1} z_{2} \cdots z_{n}\right)^{N} q\right\|^{2} \leq\|A\|^{2}\left\|\left(z_{1} z_{2} \cdots z_{n}\right)^{N} p\right\|^{2},
$$

and hence for $l>\operatorname{deg} p$,

(1) $\left\|\left(z_{1} z_{2} \cdots z_{n}\right)^{N} q_{l}\right\|^{2} \leq\|A\|^{2}\left\|\left(z_{1} z_{2} \cdots z_{n}\right)^{N} p\right\|^{2}=\|A\|^{2} \sum_{k=0}^{\operatorname{deg} p}\left\|\left(z_{1} z_{2} \cdots z_{n}\right)^{N} p_{k}\right\|^{2}$.

For each homogeneous polynomial $r=\sum_{j_{1}+\cdots+j_{n}=\mathrm{deg} r} a_{j_{1} \cdots j_{n}} z_{1}^{j_{1}} \cdots z_{n}^{j_{n}}$, an easy calculation gives

$$
\left\|\left(z_{1} \cdots z_{n}\right)^{N} r\right\|^{2}=\sum_{j_{1}+\cdots+j_{n}=\operatorname{deg} r}\left|a_{j_{1} \cdots j_{n}}\right|^{2} 2^{\operatorname{deg} r+n N}\left(j_{1}+N\right) ! \cdots\left(j_{n}+N\right) !
$$

Multiplying the two sides of (1) by $e^{n N} /\left(2^{n N} N^{l+n N+N / 2}\right)$ and letting $N \rightarrow \infty$, then applying Stirling's formula $m ! \sim \sqrt{2 \pi m} m^{m} e^{-m}$ (as $m \rightarrow \infty$ ) to (1) gives

$$
\sum_{j_{1}+\cdots+j_{n}=l}\left|a_{j_{1} \cdots j_{n}}\right|^{2}=0
$$

This means that $q_{l}=0$ for all $l>\operatorname{deg} p$, and hence the desired result follows.

We endow the polynomial ring $\mathscr{C}$ with the topology induced by the Fock space $L_{a}^{2}\left(\mathbb{C}^{n}\right)$. For an ideal $I$, we regard $I$ as module over the ring $\mathscr{C}$.

COROLlARY 2.4. Let $A: M_{1} \rightarrow M_{2}$ be a similarity. Then $A$ induces a continuous module isomorphism from $M_{1} \cap \mathscr{C}$ onto $M_{2} \cap \mathscr{C}$.

By [13, Lemma 5.2], for each ideal $I$ of the polynomial ring $\mathscr{C}$, one has $\bar{I} \cap \mathscr{C}=I$. Combining this fact with Proposition 2.1 and Corollary 2.4, we see that if $I_{1}$ and $I_{2}$ are homogeneous ideals, then a similarity $A: \bar{I}_{1} \rightarrow \bar{I}_{2}$ induces a continuous module isomorphism from $I_{1}$ onto $I_{2}$.

Let $B_{n}$ be the unit ball of $\mathbb{C}^{n}$, and $\partial B_{n}$ be the boundary of $B_{n}$. We let $\sigma$ be the unique rotation-invariant positive Borel measure on $\partial B_{n}$ for which $\sigma\left(\partial B_{n}\right)=1$. As usual, $H^{2}\left(B_{n}\right)$ denotes the Hardy space on the unit ball $B_{n}$. Let $M_{1}, M_{2}$ be invariant subspaces of $H^{2}\left(B_{n}\right)$. We say that a bounded linear operator $A: M_{1} \rightarrow M_{2}$ is a module map if $A(p h)=p A(h)$ for any polynomial $p$ and $h \in M_{1}$. 
LEMMA 2.5. If $M_{1}$ and $M_{2}$ are invariant subspaces of $H^{2}\left(B_{n}\right)$ and $A: M_{1} \rightarrow M_{2}$ is a module map, then there exists a bounded function $\phi$ on $\partial B_{n}$ such that $A(h)=\phi h$ for any $h \in M_{1}$.

PROOF. From Rudin [17], we see that all inner functions on $B_{n}$ and their adjoints generate $L^{\infty}\left(\partial B_{n}\right)$ in the weak*-topology. Set

$$
\mathscr{D}=\left\{\bar{\eta} h: \eta \text { are inner functions, and } h \in M_{1}\right\} .
$$

Then $\mathscr{D}$ is a dense linear subspace of $L^{2}\left(\partial B_{n}\right)$. We define a map $\hat{A}: \mathscr{D} \rightarrow L^{2}\left(\partial B_{n}\right)$ by $\hat{A}(\bar{\eta} h)=\bar{\eta} A(h)$. Since $A$ is a module map, the above definition is well defined. From the relation $\|\hat{A}(\bar{\eta} h)\|=\|A(h)\| \leq\|A\|\|\bar{\eta} h\|$, we see that $\hat{A}$ extends to a bounded map from $L^{2}\left(\partial B_{n}\right)$ to $L^{2}\left(\partial B_{n}\right)$. It is obvious that $\hat{A}$ satisfies $\hat{A} M_{g}=M_{g} \hat{A}$ for any $g \in L^{\infty}\left(\partial B_{n}\right)$, and hence there exists a function $\phi \in L^{\infty}\left(\partial B_{n}\right)$ such that $\hat{A}=M_{\phi}$. This insures that $A(h)=\phi h$ for any $h \in M_{1}$.

Below we prove Theorem 2.2.

ProOF. Let $A: \bar{I}_{1} \rightarrow \bar{I}_{2}$ be a similarity. Taking a homogeneous polynomial $p$ in $I_{1}$ and setting $q=A(p)$, and using [13, Lemma 5.2] and Proposition 2.3, we see that $q \in I_{2}$ and $\operatorname{deg} p=\operatorname{deg} q$. Since $\|r q\|^{2} \leq\|A\|^{2}\|r p\|^{2}$ for any homogeneous polynomial $r$, we have

$$
\left\|r q_{l}\right\|^{2} \leq\|A\|^{2}\|r p\|^{2},
$$

where $l=\operatorname{deg} q$, and $q=\sum_{s=0}^{l} q_{s}$ is the homogeneous expansion of $q$. Recall that integration in polar coordinates (corresponding the volume measure) is given by [16, page 13]

$$
\int_{C^{n}} f d v=\frac{2 n \pi^{n}}{n !} \int_{0}^{\infty} r^{2 n-1} d r \int_{\partial B_{n}} f(r \xi) d \sigma
$$

Hence from(2), we have

$$
\int_{\partial B_{n}}\left|r(\xi) q_{l}(\xi)\right|^{2} d \sigma \leq\|A\|^{2} \int_{\partial B_{n}}|r(\xi) p(\xi)|^{2} d \sigma .
$$

Since on the Hardy space $H^{2}\left(B_{n}\right)$ two homogeneous polynomials with different degrees are orthogonal, this shows that

$$
\int_{\partial B_{n}}\left|h(\xi) q_{l}(\xi)\right|^{2} d \sigma \leq\|A\|^{2} \int_{\partial B_{n}}|h(\xi) p(\xi)|^{2} d \sigma
$$


for any polynomial $h$. Let $\left[q_{l}\right]_{n}$ and $[p]_{n}$ be invariant subspaces of $H^{2}\left(B_{n}\right)$ generated by $q_{l}, p$ respectively. Then applying the preceding inequality yields the following bounded module map $B:[p]_{n} \rightarrow\left[q_{l}\right]_{n}, B p h=q_{l} h$, for each polynomial $h$. By Lemma 2.5 , there is a bounded function $f$ on $\partial B_{n}$ such that $B=M_{f}$. This implies that $q_{l}=f p$ on $\partial B_{n}$. So, $\left|q_{l}(\xi)\right| \leq\|f\|_{\infty}|p(\xi)|$ for every $\xi \in \partial B_{n}$. Since both $p$ and $q_{l}$ are homogeneous, and $\operatorname{deg} p=\operatorname{deg} q_{l}=l$, this means that for any $z \in \mathbb{C}^{n}$, $\left|q_{l}(z)\right| \leq\|f\|_{\infty}|p(z)|$. So, the function $q_{l}(z) / p(z)$ is analytic and bounded on $\mathbb{C}^{n}$, and it follows that there is a nonzero constant $\gamma$ such that $q_{l}=\gamma p$. Since $I_{2}$ is homogeneous, $q_{l} \in I_{2}$, and hence $p \in I_{2}$. The above reasoning shows that $I_{1} \subseteq I_{2}$. Note that $A: \bar{I}_{1} \rightarrow \bar{I}_{2}$ be a similarity. The same reasoning gives that $I_{2} \subseteq I_{1}$, and hence $I_{1}=I_{2}$, completing the proof.

\section{The similarity orbit of $\left[z^{m}\right]$}

From Theorem 2.2, one sees that for homogeneous quasi-invariant subspaces similarity only appears in the case of equality. Therefore, a natural problem is to determine the similarity orbit of quasi-invariant subspaces. Let $M$ be a quasi-invariant subspace. Then the similarity orbit, $\operatorname{orb}_{s}(M)$, of $M$ consists of all quasi-invariant subspaces which are similar to $M$. There is no doubt that the problem is difficult. Here we will exhibit an example to show how to determine the similarity orbit.

For a polynomial $p$, we let $[p]$ denote the closure of $p \mathscr{C}$ on the Fock space. Using sheaf theory or [11, Theorem 2.3], one easily verifies that for each $g \in[p]$ there exists an entire function $f$ such that $g=p f$. Moreover, if $p$ is homogeneous, then $[p]$ is quasi-invariant.

THEOREM 3.1. On the Fock space $L_{a}^{2}\left(\mathbb{C}^{2}\right)$, the similarity orbit orb ${ }_{s}\left(\left[z^{m}\right]\right)$ of $\left[z^{m}\right]$ consists of $[p(z)]$, where $p(z)$ range over all polynomials in the variable $z$ with $\operatorname{deg} p=m$.

Proof. Let $p(z)$ be a polynomial in the variable $z$ with $\operatorname{deg} p=m$. Then we can establish an inequality $C_{1}\left\|z^{m} f\right\|^{2} \leq\|p(z) f\|^{2} \leq C_{2}\left\|z^{m} f\right\|^{2}$ for any entire function $f$, where $C_{1}$ and $C_{2}$ are positive constants only depending on $p(z)$. In fact, One can show that there exist positive constants $C_{1}$ and $C_{2}$, which depend only on $p(z)$ such that

$$
C_{1}\left\|z^{m} g(z)\right\|^{2} \leq\|p(z) g(z)\|^{2} \leq C_{2}\left\|z^{m} g(z)\right\|
$$

for any entire function $g(z)$. Let $f=\sum_{k \geq 0} f_{k}(z) w^{k}$ be the expansion of $f$ relative to the variable $w$. Then by the equality $p(z) f=\sum_{k \geq 0} p(z) f_{k}(z) w^{k}$, we have

$$
\|p(z) f\|^{2}=\sum_{k \geq 0}\left\|p(z) f_{k}(z)\right\|^{2}\left\|w^{k}\right\|^{2} .
$$


From the above inequality we see

$$
\|p(z) f\|^{2} \leq C_{2} \sum_{k \geq 0}\left\|z^{m} f_{k}(z)\right\|^{2}\left\|w^{k}\right\|^{2}=C_{2}\left\|z^{m} f\right\|^{2} .
$$

The same reasoning gives $\|p(z) f\|^{2} \geq C_{1}\left\|z^{m} f\right\|^{2}$, and hence the required inequality is established.

Since the homogeneous quasi-invariant subspace $\left[z^{m}\right]$ is given by

$$
\left[z^{m}\right]=\left\{z^{m} f \in L_{a}^{2}\left(\mathbb{C}^{2}\right) \mid f \text { is an entire function }\right\},
$$

the preceding established inequality gives that

$$
[p(z)]=\left\{p(z) f \in L_{a}^{2}\left(\mathbb{C}^{2}\right) \mid f \text { is an entire function }\right\},
$$

and hence $[p(z)]$ is quasi-invariant.

Now we establish a map

$$
A:\left[z^{m}\right] \rightarrow[p(z)], \quad z^{m} f \mapsto p(z) f .
$$

Then by the preceding discussion and the closed graph theorem, $A$ is continuous. Obviously, $A$ is injective, surjective, and is a quasi-module map. Similarly, $A^{-1}$ also is a quasi-module map, and hence $A$ is a similarity. On the other hand, we let $M$ be quasi-invariant, and $A:\left[z^{m}\right] \rightarrow M$ be a similarity. Set $q=A\left(z^{m}\right)$. We claim that $q$ is a polynomial in the variable $z$, and $\operatorname{deg} q=m$. To prove the claim, we expand $q$ relative to the variables $w$, by $q=q_{0}(z)+w q_{1}(z)+w^{2} q_{2}(z)+\cdots$. Assume that $\operatorname{deg}_{w} q>\operatorname{deg}_{w} z^{m}=0$, here $\operatorname{deg}_{w} q$ denotes degree of $q$ in the variable $w$ (allowed to be $\infty)$. Then there exists a positive integer $s$ such that $q_{s}(z) \neq 0$. Since

$$
\left\|A\left(w^{k} z^{m}\right)\right\|^{2}=\left\|w^{k} q\right\|^{2}=\sum_{i=0}^{\infty}\left\|w^{k+i} q_{i}(z)\right\|^{2},
$$

this implies that $\left\|w^{k+s} q_{s}(z)\right\|^{2} \leq\|A\|^{2}\left\|w^{k} z^{m}\right\|^{2}$ for any positive integer $k$. Since

$$
\left\|w^{k+s} q_{s}(z)\right\|^{2}=2^{k+s}(k+s) !\left\|q_{s}(z)\right\|^{2} \quad \text { and } \quad\left\|w^{k} z^{m}\right\|^{2}=2^{k+m} k ! m !
$$

for any positive integer $k$, this clearly implies that $q_{s}=0$. This contradicts the assumption, and hence $\operatorname{deg}_{w} q=0$. So, $q$ depends only on the variable $z$. Now we expand $q$ in the variable $z$ by $q(z)=\sum_{k \geq 0} a_{k} z^{k}$. If there is a positive integer $l$, and $l>m$ such that $a_{l} \neq 0$, then the equality $A\left(z^{s} z^{m}\right)=\sum_{k \geq 0} a_{k} z^{s+k}$ implies that

$$
\left|a_{l}\right|^{2}\left\|z^{s+l}\right\|^{2} \leq\|A\|^{2}\left\|z^{m+s}\right\|^{2} .
$$


This leads to the following

$$
2^{s+l}\left|a_{l}\right|^{2}(s+l) ! \leq 2^{m+s}\|A\|^{2}(m+s) !
$$

for any positive integer $s$. This clearly is impossible, and hence $q(z)$ is a polynomial in the variable $z$ with $\operatorname{deg} q \leq m$. It is easy to see that $M=[q]$ because $A$ : $\left[z^{m}\right] \rightarrow M$ coincide on the dense set $z^{m} \mathscr{C}$ with the map $A$ considered in (3). Applying Proposition 2.3, $\operatorname{deg} q=m$. This shows that $q(z)$ is a polynomial in the variable $z$ with degree $m$. Based on the above discussion, we conclude that the similarity orbit $\operatorname{orb}_{s}\left(\left[z^{m}\right]\right)$ of $\left[z^{m}\right]$ consists of $[p(z)]$, where $p(z)$ range over all polynomials in the variable $z$ with $\operatorname{deg} p=m$.

REMARK. From the proof of Theorem 3.1, it is not difficult to see that Theorem 3.1 remains true in the case of the Fock space $L_{a}^{2}\left(\mathbb{C}^{n}\right)$ for any positive integer $n$. For $n=1$, a related problem is considered in [13].

\section{Acknowledgment}

The author is deeply indebted to the referee for numerous suggestions which made this paper more readable.

\section{References}

[1] O. Agrawal, D. Clark and R. Douglas, 'Invariant subspaces in the polydisk', Pacific J. Math. 121 (1986), 1-11.

[2] O. Agrawal and N. Salinas, 'Sharp kernels and canonical subspaces', Amer. J. Math. 109 (1987), 23-48.

[3] P. Ahern and D. Clark, 'Invariant subspaces and analytic continuation in several variables', J. Math. and Mech. 19 (1970), 963-969.

[4] S. Axler and P. Bourdon, 'Finite codimensional invariant subspaces of Bergman spaces', Trans. Amer. Math. Soc. 305 (1986), 1-13.

[5] X. Chen and R. Douglas, 'Rigidity of Hardy submodules on the unit ball', Houston J. Math. 18 (1992), 117-125.

[6] X. Chen, K. Guo and S. Hou, 'Analytic Hilbert spaces on the complex plane', J. Math. Anal. Appl. 268 (2002), 684-700.

[7] R. Douglas and V. Paulsen, Hilbert modules over function algebra, Pitman Research Notes in Mathematics 217 (Wiley \& Sons, New York, 1989).

[8] R. Douglas, V. Paulsena, C. Sah and K. Yan, 'Algebraic reduction and rigidity for Hilbert modules', Amer. J. Math. 117 (1995), 75-92.

[9] K. Guo, 'Algebraic reduction for Hardy submodules over polydisk algebras', J. Operator Theory 41 (1999), 127-138.

[10] - 'Characteristic spaces and rigidity for analytic Hilben modules', J. Funct. Anal. 163(1999), $133-151$. 
[11] —-, 'Equivalence of Hardy submodules generated by polynomials', J. Funct. Anal. 178 (2000), 343-371.

[12] _-, 'Podal subspaces on the unit polydisk', Studia Math. 149 (2002), 109-120.

[13] K. Guo and D. Zheng, 'Invariant subspaces, quasi-invariant subspaces and Hankel operators', $J$. Funct. Anal. 187 (2001), 308-342.

[14] M. Putinar, 'On invariant subspaces of several variable Bergman spaces', Pacific J. Math. 147 (1991), 355-364.

[15] S. Richter, 'Unitary equivalence of invariant subspaces of the Bergman and Dirichlet spaces', Pacific J. Math. 133 (1988), 151-156.

[16] W. Rudin, Function theory in the unit ball of $\mathbb{C}^{n}$ (Springer, New York, 1980).

[17] - New construction of functions holomorphic in the unit ball of $\mathbb{C}^{n}$, CBMS Regional Conference Series in Mathematics 63 (Amer. Math. Soc., Providence, 1986).

\section{Department of Mathematics}

Fudan University

Shanghai 200433

P. R. China

e-mail: kyguo@fudan.edu.cn 
J. Aust. Math. Soc. 75 (2003) 